\title{
Immersive technologies as a solution for general data protection regulation in Europe and impact on the COVID-19 pandemic
}

\author{
Klaudia Proniewska ${ }^{1}$, Agnieszka Pręgowska ${ }^{2}$, \\ Damian Dołęga-Dołęgowski ${ }^{1}$, Dariusz Dudek ${ }^{1,3}$ \\ ${ }^{1}$ Jagiellonian University Medical College, Krakow, Poland \\ ${ }^{2}$ Institute of Fundamental Technological Research, Polish Academy of Sciences, Warsaw, Poland \\ ${ }^{3}$ Maria Cecilia Hospital, Gvm Care and Research, Cotignola (Ra), Italy
}

\begin{abstract}
Background: General data protection regulation (GDPR) provides rules according to which data should be managed and processed in a secure and appropriate way for patient requirements and security. Currently, everyone in Europe is covered by GDPR. Thus, the medical practice also requires access to patient data in a safe and secure way.

Methods: Holographic technology allows users to see everything visible on a computer screen in a new and less restricted way, i.e. without the limitations of traditional computers and screens.

Results: In this study, a three-dimensional holographic doctors' assistant is designed and implemented in a way that meets the GDPR requirements. The HoloView application, which is tailored to run on Microsoft HoloLens, is proposed toallow display and access to personal data and so-called sensitive information of all individual patients without the risk that it will be presented to unauthorized persons. Conclusions: To enhance the user experience and remain consistent with GSPR, a holographic desk is proposed that allows displaying patient data and sensitive information only in front of the doctor's eyes using mixed reality glasses. Last but not least, it boasts of a reduction in infection risk for the staff during the COVID-19 pandemic, affording medical care to be carried out by as few doctors as possible. (Cardiol J 2021; 28, 1: 23-33)
\end{abstract}

Key words: augmented reality, mixed reality, pandemic

\section{Introduction}

The general data protection regulation (GDPR), formally called Data Protection Directive 95/46/EC, entered into force in May 2016, and European Union (EU) countries had to transpose it into their national law by 25 May 2018 [EU, 2018]. This legislation consists of 99 articles and deals with the protection of the personal data and so-called sensitive information of all individual EU citizens, and those of the European economic area inside and outside the EU community. It also protects a person and access to European or non-
-European businesses [1]. What is personal data? According to the European Commission, personal data is any information connected with a citizens private, professional, and/or public life [2]. These are, among others, data like names, surnames, photos, home address or social media activities [Article 2, Article 4(1) and (5) and Recitals (14), (15), (26), (27), (29) and (30)] [3] The second type of information, i.e., sensitive data are related to racial or ethnic origin, political opinions, religious or philosophical beliefs, genetic data, biometric data, health-related data as well as sexual orientation of individuals [Article 4(13), (14) and (15) and Article 9

Address for correspondence: Dr. Klaudia Proniewska, Jagiellonian University Medical College, ul. Św. Anny 12, 31-008 Kraków, Poland, tel. +48 793060785, e-mail: klaudia.proniewska@uj.edu.pl 
and Recitals (51) to (56)] [4]. GDPR is similar to United States (US) privacy law and Federal Trade Commission settlements with companies, but is more restrictive. The impact of GDPR on health services and clinical research on a human subject is, therefore, essential in all aspects [5]. Doctors are obliged to comply with its procedures from information to patients to data sharing requirements. The general principles that should be applied to patients' data are described in Article 5. All personal data of subjects should be available only for a chosen doctor. This is a huge technological challenge. Still, in doctor practices, one can find desks and files full of different types of documentation, books, patient data, and other information. Digitalization is an evolving field and progresses continually, but there is still much to do. An increasing number of doctors have been using this data in their exam rooms, on their tablets, PC's and laptops. All of those devices have the same limitation, a finite amount of information that can be displayed at the same time. Besides that, in most of the cases, a screen with restricted details can be viewed by anyone in the examination room.

Immersive technologies such a virtual reality (VR), augmented reality (AR), and mixed reality (MR) join the real world with a computer-generated one providing a composite view [6]. AR is an enhanced version of the physical world through the use of different stimuli like audio or vision. Applying additional elements to three-dimensional (3D) virtual images should be happening in real-time, be interactive, and enable users, who usually wear a head-mounted display, free movement in 3D displays [7, 8]. Mixed reality is a hybrid of both augmented and virtual realities. AR/VR/MR devices offer a new quality for art and entertainment [9, 10], maintenance [11, 12], architecture [13], industry [14] healthcare delivery and education in medicine [15]. Recently, the number of applications in the healthcare sector have systematically increased. In Tepper et al.'s study [16], augmented reality was used in the operating room to improve the decision-making process and improve the surgeon workflow. It is also increasingly applied in the education sector, like anatomy [17], nursing $[18,19]$, and neurology [20] courses. AR-based clinical experience allows visually assessing both the external form and an internal organ structure. To introduce this new technique, it is necessary to use specific devices, i.e., head-mounted displays, and various sensors, which will be applied in the diagnosis process [21,22]. Thus, according to GDPR provisions, holograms and mixed reality devices like Microsoft HoloLens [23] or HTC
VIVE Pro [24] can be the right solution. These technologies create an opportunity to remove everything from the doctor's desk except for MR glasses. All personal data are made available only to be seen by said doctor.

This paper aims to find a solution, which follows the 95/46/EC EU directive in the field of medicine. Here, a proposed holographic application (HoloView), that is tailored to run on Microsoft HoloLens [23]. The HoloView application unables users (doctors) to display all information needed about their subjects' health status. Moreover, it gives users a wide range of views. The users felt like they were working on three normal LCD curved screens connected by edges. The biggest plus is that all pieces of information are visible only to the doctor and no one else. The device is secured with built-in monitor whichchecks if the device is taken off of the doctor's head - which results in blocking access to the application. Thus, the proposed approach contributes to personal data security and so-called sensitive information protection.

Additionally, the subject of this project is to develop knowledge on the use of HoloView in medical education at all levels during the COVID-19 pandemic. The proposed solution presents new possibilities and enables additional support in the process of clinical (pre-procedural) diagnostics during medical procedures (intraoperative). The simulation of medical procedures through the Mixed Reality HoloView system offers an opportunity to train medical students, paramedics, and doctors across a range of specialists in a safe (risk-free), realistic and repeatable environment. It eliminates harmful consequences to patients and offers repeatable procedures. The system consists of four main components, i.e. central indirect server, an artificial intelligence module based on deep neural networks, a wireless health check sensors platform, and the MR application (HoloView COVID pandemic). The central indirect server is responsible for independent work and all types of communication with external units, digital sensors, and devices. It does an analysis of subject data, classifications, potential diagnoses, and an artificial intelligence-based software pertaining to treatment. The digital diagnostic sensors module allows the performance of health checks on patients.

\section{Methods}

\section{Immerse technologies}

Augmented reality extends a real environment with computer-generated information using differ- 


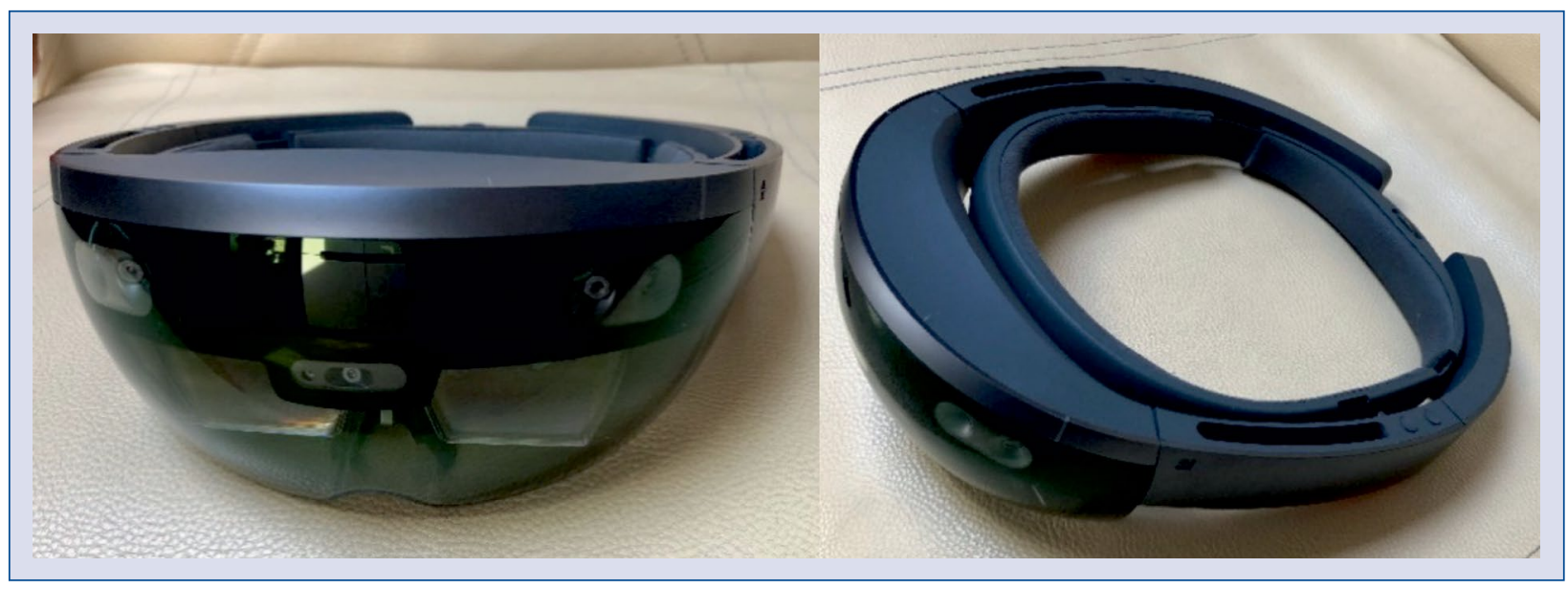

Figure 1. Microsoft HoloLens.

ent stimuli like audio or vision, rather than replacing the physical world as with VR. It integrates with the users and allows them to manipulate the digital data in real-time and allowing users to manage these data [7, 8]. In 2016, the game called Pokemon Go, which is one of many successful mobile games in the world, was realized using AR technologies and were thus raised to public attention [25, 26]. The number of AR/VR/MR applications in the field of medicine has been increasingly growing since doctors want to give better outcomes for their patients [15]. HoloLens from Microsoft [23] and VIVE Pro from HTC [24] are two commercially available sets for Mixed Reality. First, one is an independent device and does not require a separate operation space or manual controllers. It is fully integrated with Microsoft Enterprise systems; its interface is known to users, which are familiar with using the Windows operating system on other computer platforms. The disadvantage of Microsoft's solution is that the commercial license for HoloLens has a much higher price than VIVE Pro (even taking into account the cost of an additional workstation) [27].

In terms of testing innovative technological solutions, as well as their regular use in the diagnosis, therapy, and rehabilitation of patients, medicine is the leading discipline. In this area, the most practical and promising innovation is exactly the application of Mixed Reality, i.e., the combination of the image of real objects and biological signals with the data obtained, e.g., during the diagnostic process using imaging techniques. It is also predicted that VR, AR, and MR technologies will have a significant impact on the development of telemedicine.

\section{Microsoft HoloLens}

During the past few years, thanks to the miniaturization process. In 2016 the Microsoft Corporation released a product called "HoloLens" (Fig. 1) [23]. A device in the form of goggles with a built-in battery, microcomputer, cameras, microphone, speakers, and holographic projectors. A user wears HoloLens, which is in the form of big glasses. Build-in micro projectors generate an image, in fact, a hologram, onto the glasses in front of the user's eyes. HoloLens functionality does not end with only displaying a flat image. The $3 \mathrm{D}$ imaging occurs when an active mapping of the environment is used, which fully recognizes the shapes of objects and terrain, in whose area users are moving. Besides that, depth cameras analyze the entire environment around users like tables, chairs, walls, etc. A microcomputer analyzes the shape of the room and decides about a wayto display a hologram in front of the user in a manner compatible with that environment. For example, when placed on a table or displayed on screens on the walls, so the view becomes more natural to the operator using the goggles.

Microsoft HoloLens is the only product that provides interaction: eye-tracking, service using gestures, and voice support. This device is completely autonomous (no connection to a computer or phone to work is needed). It is light, robust and looks very aesthetically pleasing. Depending on the software, the device can display an image in hologram form for both VR and AR.

The first generation of HoloLens glasses were based on a quad-core Intel processor Atom x5-Z8100 clocked at $1.04 \mathrm{GHz}$. This is a 64-bit 


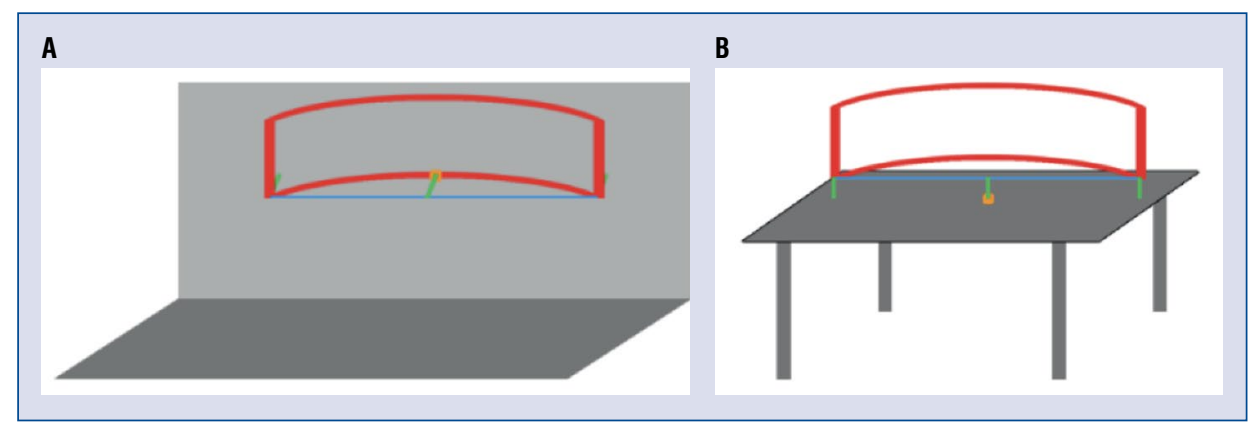

Figure 2. Schemat of the information/images displayed by HoloLens; A. On the wall; B. On the desk.

unit made in the technological process of $14 \mathrm{~nm}$. It was supported by HoloLens Graphics, which also was prepared by Intel. The first generation was equipped with $2 \mathrm{~GB}$ of RAM. HoloLens 2 (second generation) is equipped with a system displaying information in $2 \mathrm{~K}$ resolution and a 3:2 aspect ratio. Data projection adapts to the operator's eyes, socalled positioning the image relative to eyes-position in 3D. The device has an image sensor (Azure Kinect sensor), an accelerometer, a gyroscope, and a magnetometer. The built-in 8MP camera allows recording movies in $1080 \mathrm{p}$ and $30 \mathrm{fps}$ quality. HoloLens 2 also has five channel microphones and advanced tracking systems for both hands (fully recognizing all hand movements, including individual fingers). The Qualcomm Snapdragon 850 processor performs the whole operation. The device provides $802.11 \mathrm{ac}$ wireless connectivity $2 \times 2$ a Bluetooth version 5 , modules and sensors: inertial measuring unit, four cameras for recognition of the surroundings, one camera for measuring depth, a light sensor and four microphones. For recording video in $30 \mathrm{fps}$, two photographic cameras are available: the first $2.4 \mathrm{Mpx}$ and the second $1.1 \mathrm{Mpx}$. The second generation also has bigger storage, a $64 \mathrm{~GB}$ data carrier (for the user, just over $54 \mathrm{~GB}$ is available). It works under the control of the Windows 10 operating system in a 32-bit version. A 16.5 Wh powers it entirely.

In Figure 2, the display method using HoloLens is presented. The HoloLens area of view is schematically indicated by a red area (red window). Before information/images are displayed, the dedicated application needs to be provided a desired working space. The pointer marked schematically as an orange point, is responsible for this activity (Fig. 3). It knows on which kind of environment the information/images will be displayed on the HoloLens. The whole procedure is done by scanning the environment around the pointer in $3 \mathrm{D}$. The pointer

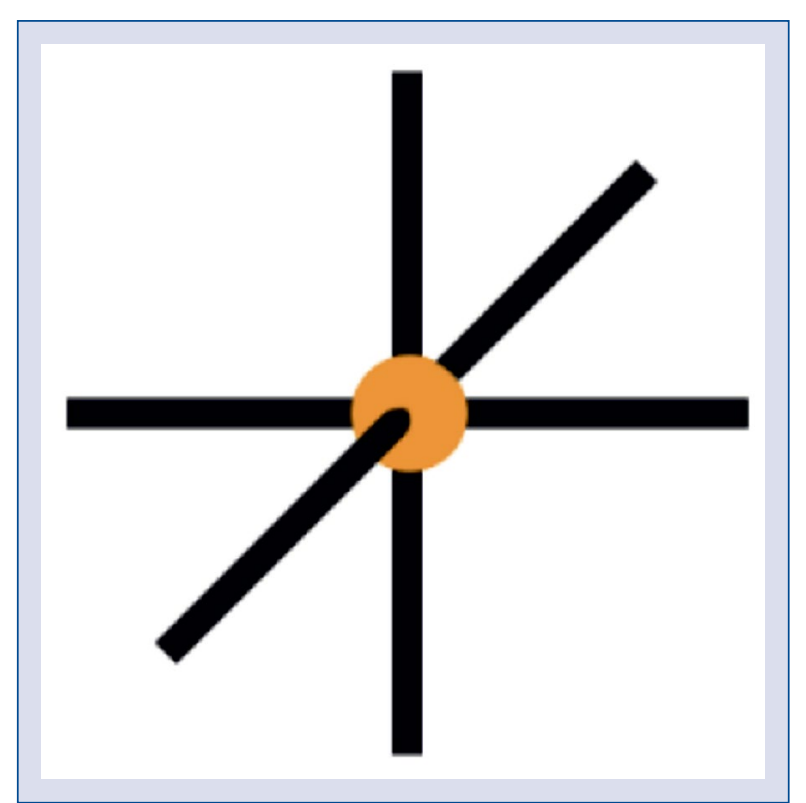

Figure 3. Schematic scan of the environment by the pointer.

performs a two-stage scanning process. In the first step, it scans the mapped environment above and below. If the orange point does not create a vertical line, it means that the user is pointing at a wall (Fig. 2A). The second check is responsible for horizontally checking the mapped area. If checkedpoint creates a horizontal line, it means that the user is on the table, and the application should be displayed on a horizontal space (Fig. 2B). The main difference between $A$ and $B$ cases is that when the user shows a hologram on the wall, he/she does not want a situation where some of the displayed data will be hidden inside the wall so it will not be visible. Because the proposed application, i.e., HoloView, has a semicircular shaped orange pointer to change the way the hologram projects. If the 
pointer is on a wall, it displays HoloView where the middle starts from the pointer schema as presented in Figure 2B. If it is displayed on a desk, the orange pointer is in the middle of the line created by both corners of HoloView shape (Fig. 2A). In the case of holograms on a desk, it displays right away, and can interfere with stuff on the desk, which is also unwanted.

\section{AR/MR application}

In recent years, the first devices using holographic technologies have already appeared on the market. Although they differ in image quality, size, weight, and even performance, advances in this technology are visible, and subsequently improved versions or prototype devices show that it is becoming a realistic-looking hologram and will improve over the next few years. The current quality of holograms already allows its effective application in everyday life [28]. In El-Schich et al. [29], the cancer cells using computer-generated holography were analyzed. An interesting application of holographic technology incoherent imaging was presented in Wang et al. [30]. Also, the off-axis digital holography shown in Claus et al. [31] seems to be a promising solution in intraoperative medical imaging. The designing and implementation of $\mathrm{AR} / \mathrm{MR}$ applications and the flat screens of computers or tablets differ very much. The first one has more control over processes and their design. $\mathrm{AR} / \mathrm{MR}$ devices in the form of head-mounted display works with the human senses and human preferences which are varied depending on the unit. In the case of HoloLens [23] and application design, it is possible to choose the way of design using voice commands or gestures. Also, the $3 \mathrm{D}$ user AR/MR-based interfaces are a huge challenge. Commonly used two-dimensional (2D) interfaces have many buttons, windows, cards, and visual options available in a $2 \mathrm{D}$ space. The development of a 3D graphical interface that engages the user visually and has an emotional meaning is an essential part of the design process, in particular to medical aspects. In Aruanno et al. [32], MemHolo, i.e., the prototype of the first HoloLens application designed for a subject suffering from Alzheimer's disease, was presented. Users can practice shortterm and spatial memory with it in a controlled virtual environment. In turn, in McDuff et al. [33] system, CardioLens to the physiological measurement of multiple people is shown. CardioLens is a MR application running on Microsoft devices, allows noncontact measurement of the heart rate.

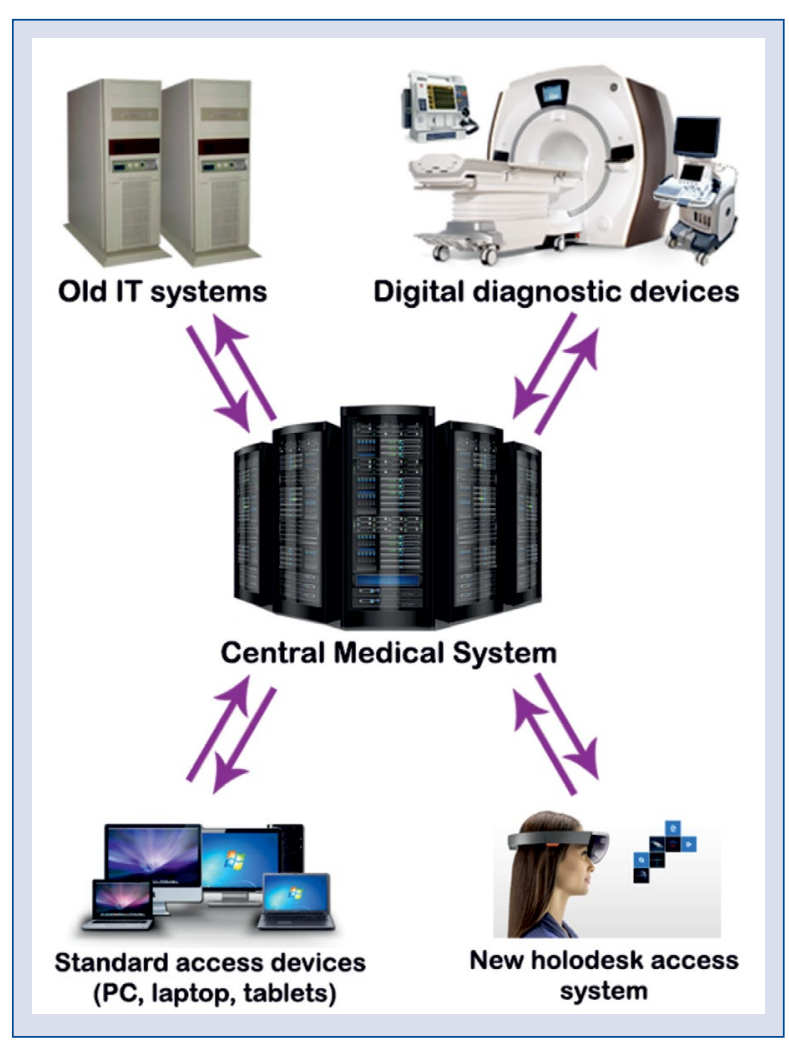

Figure 4. Holographic assistant for doctors (HoloView COVID-19 pandemic assistant for doctors).

\section{Results and Discussion}

The general idea of the proposed holographic assistant for a doctor is widely presented in Proniewska et al. [34] and Figure 4. The system consists of four main components, i.e., central indirect server, artificial intelligence module based on deep neural networks, a wireless health check sensors platform, and an MR application (HoloView). The central indirect server is responsible for independent work and all types of communication with external units, digital sensors, and devices. Analysis of a subject's data, classifications, potential diagnoses, and treatment is done by the artificial intelligence-based software. The digital diagnostic sensor module allows for the performance of health checks on patients. HoloView works as a front-end application. The software engineering splits application design into two separate parts: front-end and back-end. The most straightforward example in understanding this can be applied where all data manipulation and storage are placed on the server. This can be called "back end", the entire processing is done behind the user's eyes. There should also 


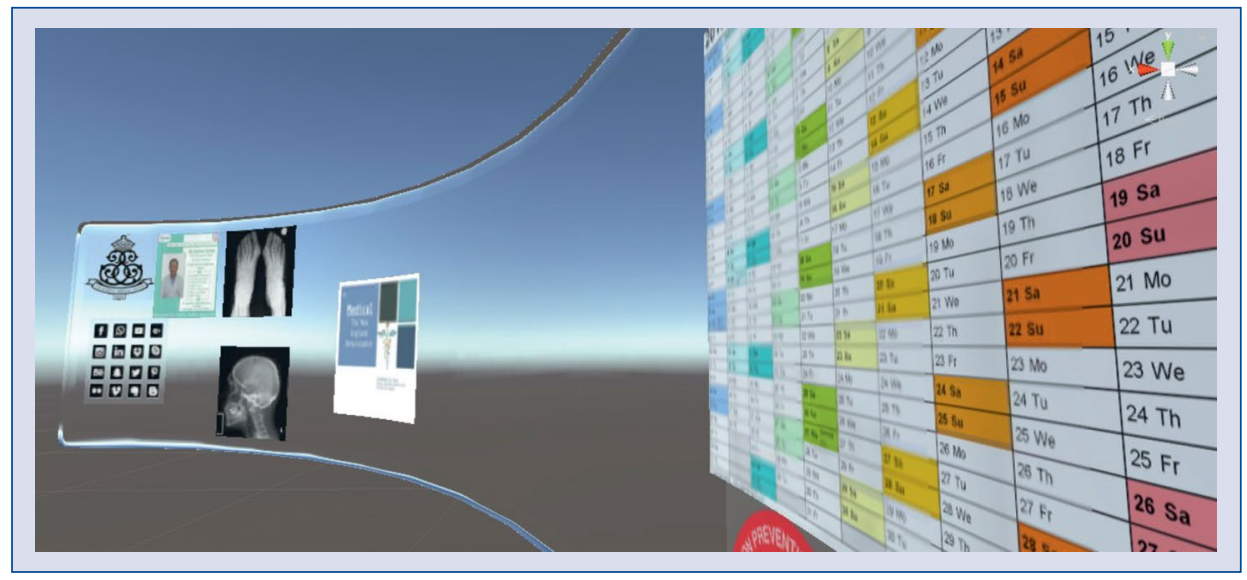

Figure 5. The perspective of the HoloView application as seen through user's eyes.

be an application-client which allows viewing data from a server, sending commands to manipulate it and display everything in a presentation layer ina way that a user will be able to see data most simply and understandably. This is called the front-end.

The proposed application is designed according to a rule that a user with a client-server connection over HTTP post/gets commands which automatically download and send data from the server. Here, the data are stored as an XML file. HoloView can adapt to any existing client-server infrastructure. Adaptation of the server has only one requirement, i.e., use of the data in an XML format. The dedicated server location is set up statically on the application. The presentation of the data displayed using the HoloLens device is a big challenge. All information should be given in the most attractive way so users will accept this without the related stress in changing his habits related to using the $3 \mathrm{D}$ application instead of $2 \mathrm{D}$ applications. HoloView application utilizes multiple curved screens on a user's desk (Fig. 5). The user, wearing HoloLens glasses, feels like he/she is using three normal LCD curved screens connected by edges. But there are crucial differences that make the HoloLens device with it's dedicated application a much better solution. First, the unused working space on the user field of view disappears. It can be compared to taking away everything from the desk and not using an LCD screen. In cases where this space is needed again, it is enabled automatically. Secondly, if someone is using spacial mapping to display information it happens in just a few seconds, goggles scan the environment around them and create the space/room in their memory model. The user then decides in which place they would like to see the information. HoloLens device displays data which looks like they were developed specifically for that user's environment. This is made possible owing to the use of spatial mapping, which helps to show the script. It allows deciding in which form, what way, what size, data should be displayed to fill the entire available space.

To make the hologram "right", it is important to examine a few aspects in developing the application. The main challenge in creating an AR/MR application is to understand so-called spatial mapping, which is a digital representation of the real world. To design a spatial awareness system, the Microsoft Mixed Reality Toolkit (MRTK) was used [35]. This is a cross-platform for building AR, MR, and VR. It offers functions like "ToolTip", which enables creating a user interface annotation with the line that points somewhere on the 3D object. Another interesting function is "Manipulation Handler", which allows one to "take" a 3D object and rotate it as with a hand manipulating a physical object in reality. HoloLens 2 has a new function, i.e., eye-tracking, which allows mapping points where an operator looks. This could be used with an intuitive zoom functionality. The MRTK platform scans the environment around the HoloLens glasses while the users move their heads. During the scanning process, the memory device inside, the HoloLens creates a 3D model of the space. In the next step, this model can be used to generate behaviors and interactions as realized in the real world. For example, when a ball is rolling on a table and reaches the edge of the table, it will fall and change its position to the floor level. Another critical issue with designing and implementing $\mathrm{AR} / \mathrm{MR}$ applications is hologram placement, i.e., selection 

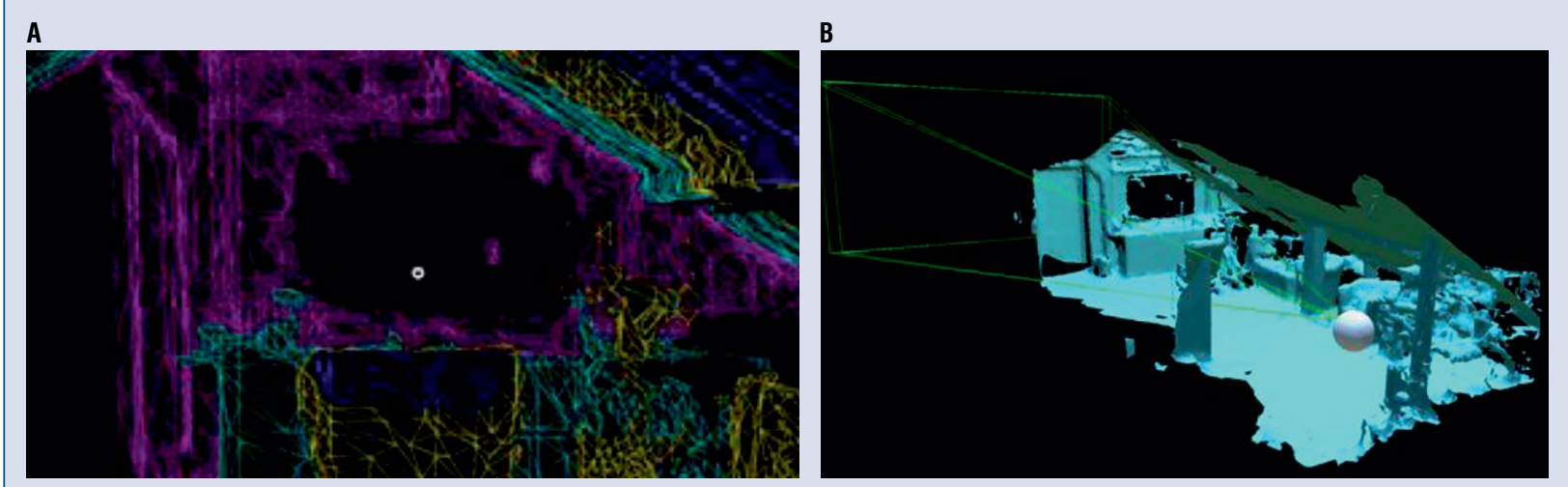

Figure 6. Images of the mapping process; A. The mapped environment with HoloLens position; B. Operator's perspective view.

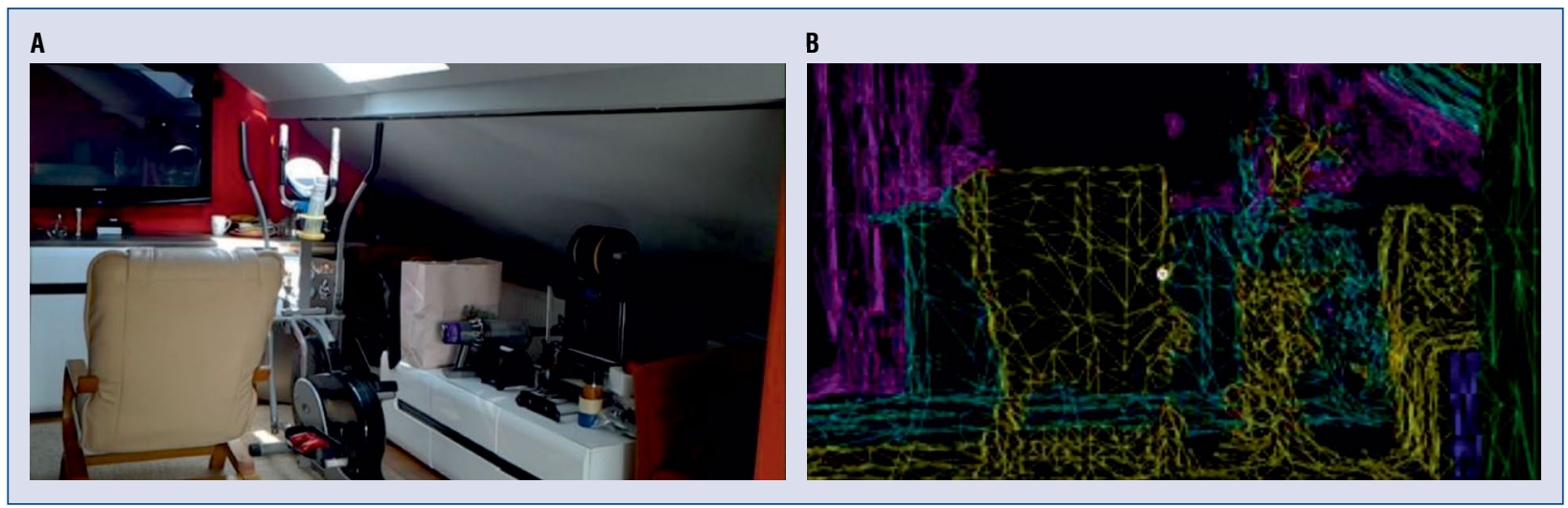

Figure 7. Spatial mapping mesh trough the HoloLens covering a room; A. Photo was taken with a camera built into the goggles; B. Shot of the scanned space.

of a spatial location. To avoid losing a sense of control by users, a specially mapped model of the environment to place and affix generated holograms in the space required. This is important when wanting to see a 3D model locked in space, which stays in the same place. For example, when the model of a box is generated, which is later placed on a table, this box should stay exactly in one place. Even when we walk around the table, it should remain in the same position on the table. An understanding of occlusion is also required. The application should also react to a specially mapped environment in a certain way, i.e., provide additional visual feedback. A good example can be a virtually displayed LCD screen on a table. When we place it in front of this generated model, for example, a real laptop, the screen of our model should react in the right way. In some cases, the users can interact with the holograms, and occlusion is not necessary. Of course, AR/MR would not be the same without physical reactions. When creating a rubber ball model that fell from the table, it is expected to bounce on the floor. A different reaction is that which is desired from a glass cup, which should break into pieces.

In Figure 6A, the mapped environment (attic room) using HoloView is shown. The location of the HoloLens, i.e., white ball relative to the room and the direction of view, its angle, and area that HoloLens view covers, marked by green lines, are illustrated. It is a model generated inside the HoloLens system that also serves to determine the response of models/holograms relative to the environment. The same scene from an operator's point of view is presented in Figure 6B. This frame is indicated by the green lines, which are visible in Figure $6 \mathrm{~A}$. The mesh reflects scene geometry by surface, which can be filtered and textured after the scanning process.

In Figure $7 \mathrm{~A}$ a real photo of the mapped frame is presented while in Figure $7 \mathrm{~B}$ corresponding 


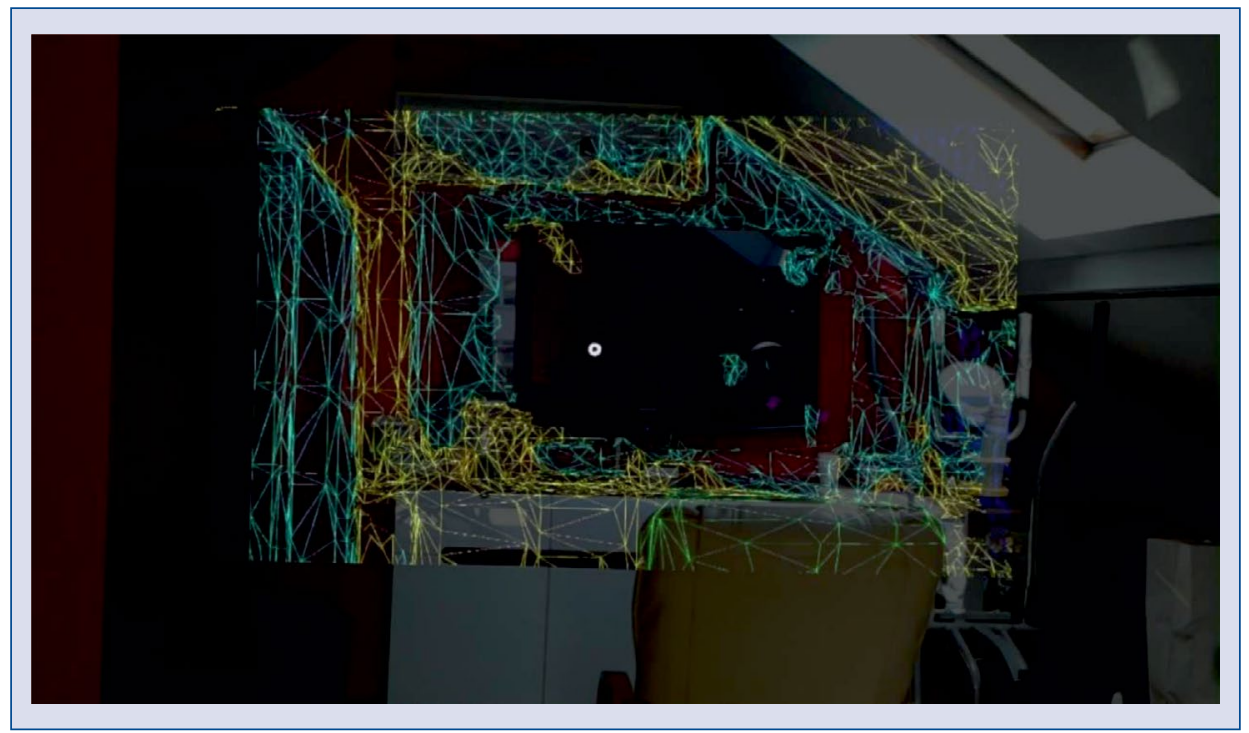

Figure 8. The limited vision range using the first generation of the Microsoft goggles.
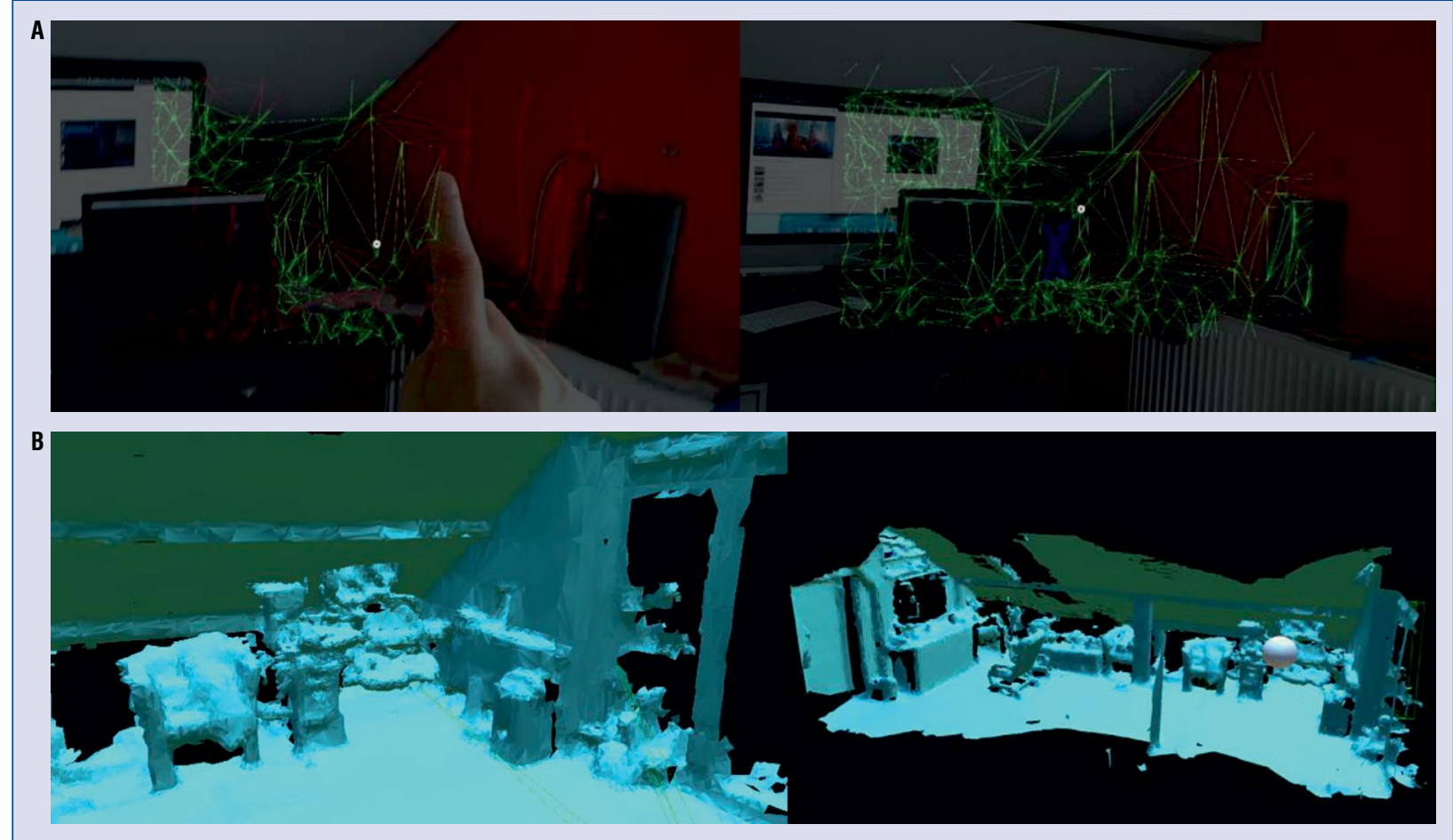

Figure 9. Shot of a corner of the room using HoloLens; A. Spatial mapping; B. Mapped area.

scanned space is shown. A view of the HoloLens with the spatial map of activity is visualized in Figure 8 . The limited range of vision offered by the first generation of the goggles is visible, i.e., only part of the image has an overlaid spatial mapping. The spatial mapping of the room is also shown in
Figure 9A, while in Figure 9B a mapped environment marked by the white ball position of the HoloLens is presented.

Thus, the HoloLens changes the way that information can be perceived, i.e., doctors' documentation working space. The proposed solution 


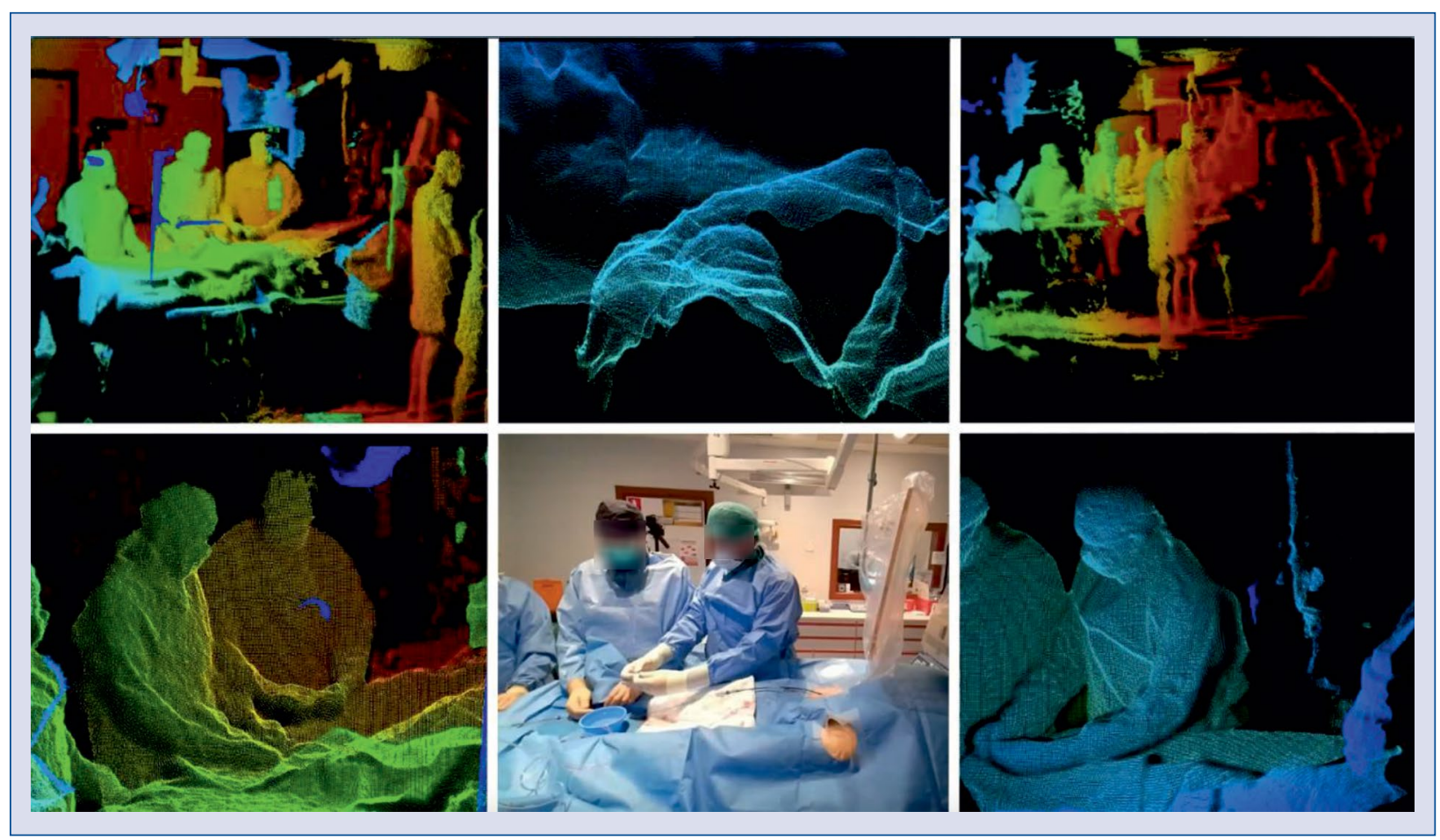

Figure 10. Experimental validation of the HoloView application.

removes almost everything from the doctor's desk. No cables, monitors, or even a mouse or keyboard is needed. Thanks to holographic technology, multiple screens can be used around a doctor's desk. A number of them can be selected in the way that doctors like. One huge screen or maybe four smaller screens with different pieces of information on each of them. Doctors decide exactly what is needed at that moment. It could be an RTG photo, treatment history, or maybe a schedule to plan the next visit. Everything becomes available right in front of the doctor. All information is displayed in front of the doctor to remind them about important facts regarding the subject like a dangerous reaction for anesthetics, heart problems, or HIV, AIDS sickness as when doctors should engage with high caution. Everything becomes available only after the doctor types a password on a virtual keyboard hanging in the air.

The impetus of digital technologies have entered almost every area of life, and so too, are hackers who are looking for innovative ways to obtain personal data. Severe financial penalties imposed on entities responsible for the leakage of personal data of their users or clients, which were introduced in May last year together with the EU regulation on the protection of personal data, which force companies and universities to control the processing of data carefully, especially in pandemic times. Global corporations, but also small enterprises are required to increase their levels of security. It is worth remembering, however, that even GDPR realization is not sufficient in protecting us from potential threats, so it is crucial to be aware of how to care for information available on the Internet and minimize the dangers present on the Internet.

The system proposed has also been validated by doctors under real conditions, i.e., in the operating room (which is shown in Fig. 10). The cardiologists from University Hospital in Krakow carried out the patent for amen ovale structural procedure using HoloView.

General data protection regulation [1] allows for broadening patient confidentiality and medical data protection. Changes in the law, especially in the sector of medical research, are discussed in Rumbold et al. [36]. Simulation on policy effecting the Digital EU Public Health Sector was presented in Yuan et al. [37]. The main advantage of the proposed HoloView solution is that the displayed data is only visible to a person wearing HoloLens goggles. Owing to that, additional confidence is gained as no one else can see restricted data. The patient is recognized using an existing $\mathrm{QR} /$ barcode. Access to the application is possible after entering 
a password on the virtual keyboard by the doctor. Patient personal and sensitive information like names, addresses, health status is presented only to the eyes of a doctor who wears a head-mounted display. A safe Internet connection is the only further requirement for security

Another critical issue is connected with Global Health Security [38]. This is dependent upon having an adequately prepared and healthy security workforce which is necessary to ensure the safety of medical service during and after the pandemic of COVID-19. Kochanczyk et al. [39] proposed a Susceptible-Infected-Infectious-Excluded model to analyze the influence of contact rate on the dynamics of COVID-19. It turned out that the pandemic can be limited by limiting contact availability. This can be done during a medical consultation using the HoloView application. Augmented technology has also been tested during COVID-19 with teaching and learning, particularly in medical education $[40,41]$. The proposed solution protects the doctor by providing a safe distance to the patient. The future remains unknown, but the pandemic can be an enduring transformation in medicine and education with the application of immersive technologies [42].

\section{Conclusions}

Changes in European law have had a huge influence on the way in which organizations handle and manage user data and sensitive information. One can expect the requirement to inform users what information about them will be used for and it is essential to protect these data. Even US healthcare units additionally have to abide by the Health Insurance Portability Accountability Act when they want to collaborate with European organizations. The development and implementation of provisions protecting us against cybercrime is a long-term process requiring the involvement of both European and national legislators and dialogue with entities from various sectors of the economy, including health protection organizations. Given the scale and complexity of cybercrime, it is necessary to develop an interdisciplinary system of specialist provisions, covering primary regulatory and personal data protection provisions. The proposed solution based on immersive technologies, such as holography and MR, meet the requirements imposed by GDPR regulations. It removes everything from a doctors' desk and is replaced with MR glasses, which are protected by a password for each doctor. Information about patients are processed and visualized in the Microsoft HoloLens which is placed on the head. Thus, all health data are visible only to the doctor; no other person present in the room has access to this data. Another advantage of the proposed solution is to ensure a safe distance between doctor and patient. The use of the Holoview during the COVID-19 pandemic simplifies documentation. It ensures continued high-quality care in times of a significant increase in the number of patients (including critical patients), where specialists/health care providers or other specialist colleagues can be involved in patient care without actually being present.

\section{Acknowledgements}

This paper has been conceived through participation in Jagiellonian University Collegium Medicum.

\section{Funding}

The publication of this article was funded by the Priority Research Area qLife under the program "Excellence Initiative - Research University" at the Jagiellonian University in Krakow (application number 06/IDUB/2019/94).

\section{Conflict of interest: None declared}

\section{References}

1. General Data Protection Regulation. Regulation (EU) 2016/679 of the European Parliament and of the Council of 27 April 2016 on the protection of natural persons with regard to the processing of personal data and the free movement of such data, and repealing Directive 95/46. Official Journal of the European Union, 2016, 59, 1-88.

2. Hoofnagle C, Sloot Bv, Borgesius F. The European Union general data protection regulation: what it is and what it means. Information Communications Technology Law. 2019; 28(1): 65-98, doi: 10.1080/13600834.2019.1573501.

3. European Commission Law. https://ec.europa.eu/info/law/lawtopic/data-protection/reform/what-personal-data_en (accessed on 14 February 2020).

4. European Commission Data Protection. https://ec europa eu/info/ law/law-topic/data-protection en (accessed on 14 February 2020).

5. European Commission Data Protection. https://ec europa eu/info/ law/law-topic/data-protection/reform/rules-business-and-organisations/legal-grounds-processing-data/sensitive-data/what-personaldata-considered-sensitive_en (accessed on 14 February 2020).

6. Suh A, Prophet J. The state of immersive technology research: A literature analysis. Comp Hum Behavior. 2018; 86: 77-90, doi: 10.1016/j.chb.2018.04.019.

7. Azuma R. A Survey of Augmented Reality. Presence: Teleoperators Virtual Environments. 1997; 6(4): 355-385, doi: 10.1162/ pres.1997.6.4.355.

8. Azuma R, Baillot Y, Behringer R, et al. Recent advances in augmented reality. IEEE Computer Graphics Applications. 2001; 21(6): 34-47, doi: 10.1109/38.963459. 
9. Ozbek SC, Giesler B, Dillmann R. Jedi training: playful evaluation of head-mounted augmented reality display systems. The Conference Medical Imaging, Proceedings of SPIE, San Diego, California, USA, 2004.

10. Lee J, Kim Y, Heo MH, et al. Real-Time projection-based augmented reality system for dynamic objects in the performing arts. Symmetry. 2015; 7(1): 182-192, doi: 10.3390/sym7010182.

11. Schwald B, Laval B. Anaugmentedrealitysystemfortrain-ing and assistance to maintenance in the industrial context. Proceedings of the International Conference in Central Europe on Computer Graphics, Visualization and Computer Vision, 425-432, University of West Bohemia, Plzen, Czech Republic, 2003.

12. Ferraguti F, Pini F, Gale T, et al. Augmented reality based approach for on-line quality assessment of polished surfaces. Robotics Computer-Integrated Manufacturing. 2019; 59: 158-167, doi: 10.1016/j.rcim.2019.04.007.

13. Grasset R, Decoret X, Gascuel JD. Augmented reality collaborative environment: calibration and interactive science editing. Proceedings of the Virtual Reality International Conference (VRIC '01), Laval Virtual, 2001.

14. Kumar GA, Kumar Patil A, Kang TW, et al. Sensor fusion based pipeline inspection for the augmented reality system. Symmetry. 2019; 11: 1325.

15. Eckert M, Volmerg JS, Friedrich CM. Augmented Reality in Medicine: Systematic and Bibliographic Review. JMIR Mhealth Uhealth. 2019; 7(4): e10967, doi: 10.2196/10967, indexed in Pubmed: 31025950.

16. Tepper OM, Rudy HL, Lefkowitz A, et al. Mixed reality with HoloLens: where virtual reality meets augmented reality in the operating room. Plast Reconstr Surg. 2017; 140(5): 1066-1070, doi: 10.1097/PRS.0000000000003802, indexed in Pubmed: 29068946.

17. Keenan ID, Ben Awadh A. Integrating $3 D$ visualisation technologies in undergraduate anatomy education. Adv Exp Med Biol. 2019; 1120: 39-53, doi: 10.1007/978-3-030-06070-1_4, indexed in Pubmed: 30919293.

18. Foronda CL, Alfes CM, Dev P, et al. Virtually nursing: emerging technologies in nursing education. Nurse Educ. 2017; 42(1): 14-17, doi: 10.1097/NNE.0000000000000295, indexed in Pubmed: 27454054.

19. Hauze SW, Hoyt HH, Frazee JP, et al. Enhancing nursing education through affordable and realistic holographic mixed reality: the virtual standardized patient for clinical simulation. Adv Exp Med Biol. 2019; 1120: 1-13, doi: 10.1007/978-3-030-06070-1_1, indexed in Pubmed: 30919290.

20. Procházka A, Vyšata $\mathrm{O}$, Charvátová $H$, et al. Motion symmetry evaluation using accelerometers and energy distribution. Symmetry. 2019; 11(7): 871, doi: 10.3390/sym11070871.

21. Evans L, Taubert M. State of the science: the doll is dead: simulation in palliative care education. BMJ Support Palliat Care. 2019; 9(2): 117-119, doi: 10.1136/bmjspcare-2018-001595, indexed in Pubmed: 30254018.

22. Wang S, Parsons M, Stone-McLean J, et al. Augmented reality as a telemedicine platform for remote procedural training. Sensors (Basel). 2017; 17(10): 2294, doi: 10.3390/s17102294, indexed in Pubmed: 28994720.

23. Microsoft HoloLens. https://www microsoft com/en-us/hololens (accessed on 14 February 2020).

24. HTC VIVE Pro. https://www vive com/us/product/vive-virtual (accessed on 14 February 2020).

25. Fobes. https://www forbes com/sites/jvchamary/2018/02/10/ pokemon-go-science-health-benefits/\#41114f2c3ab0 (accessed on 14 February 2020).
26. Marquet O, Alberico C, Hipp AJ. Pokemon GO and physical activity among college students. A study using Ecological Momentary Assessment, Computers in Human Behavior. 2018; 81: 215e222.

27. Ogdon D. HoloLens and VIVE Pro: Virtual Reality Headsets. J Med Library Association. 2019; 107(1), doi: 10.5195/ jmla.2019.602.

28. Corda R, Giusto D, Liotta A, et al. Recent advances in the processing and rendering algorithms for computer-generated holography. Electronics. 2019; 8(5): 556, doi: 10.3390/electronics8050556.

29. El-Schich Z, Mölder AL, Wingren AG. Quantitative phase imaging for label-free analysis of cancer cells - focus on digital holographic microscopy. Applied Sciences. 2018; 8(7): 1027, doi: 10.3390/app8071027.

30. Wang H, Dong Z, Fan F, et al. Characterization of spatial light modulator based on the phase in fourier domain of the hologram and its applications in coherent imaging. Applied Sciences. 2018; 8(7): 1146, doi: 10.3390/app8071146.

31. Claus D, Hennenlotter J, Liting Qi, et al. Variable wavefront curvature phase retrieval compared to off-axis holography and its useful application to support intraoperative tissue discrimination. Applied Sciences. 2018; 8(11): 2147, doi: 10.3390/app8112147.

32. Aruanno B, Garzotto F. MemHolo: mixed reality experiences for subjects with Alzheimer's disease. Multimedia Tools Applications. 2019; 78(10): 13517-13537, doi: 10.1007/s11042-018-7089-8.

33. McDuff D, Hurter C, Gonzalez-Franco M. Pulse and vital sign measurement in mixed reality using a HoloLens. Proceedings of the 23rd ACM Symposium on Virtual Reality Software and Technology. 2017; Göteborg, Sweden, 34,, doi: 10.1145/3139131.3139134.

34. Proniewska K, Dołęga-Dołęgowski D, Dudek D. A holographic doctors' assistant on the example of a wireless heart rate monitor. Bio-Algorithms Med-Systems. 2018; 14(2), doi: 10.1515/ bams-2018-0007.

35. Microsoft MRTK. https://microsoft github io/MixedRealityToolkit-Unity/Documentation/GettingStartedWithTheMRTK html (accessed on 20 August 2019).

36. Rumbold JM, Pierscionek B. The effect of the general data protection regulation on medical research. J Med Internet Res. 2017; 19(2): e47, doi: 10.2196/jmir.7108, indexed in Pubmed: 28235748.

37. Yuan B, Li J. The Policy Effect of the General Data Protection Regulation (GDPR) on the Digital Public Health Sector in the European Union: An Empirical Investigation. Int J Environ Res Public Health. 2019; 16(6), doi: 10.3390/ijerph16061070, indexed in Pubmed: 30934648.

38. French A. Simulation and modeling applications in global health security. Global Health Security. 2020: 307-340, doi: 10.1007/978-3-030-23491-1_13.

39. Kochanczyk M, Grabowski F, Lipniacki T. Dynamics of COVID-19 pandemic at constant and time-dependent contact rates. 2020.

40. Ahmed H, Allaf M, Elghazaly H. COVID-19 and medical education. Lancet. 2020.

41. Badwan B, Bothara R, Latijnhouwers M, et al. The importance of design thinking in medical education. Med Teach. 2018; 40(4): 425-426, doi: 10.1080/0142159X.2017.1399203, indexed in Pubmed: 29125007.

42. Hollander JE, Carr BG. Virtually Perfect? Telemedicine for Covid-19. N Engl J Med. 2020; 382(18): 1679-1681, doi: 10.1056/ NEJMp2003539, indexed in Pubmed: 32160451. 